\title{
Amazing Properties of Fractal Figures
}

\section{Mandrazhy Oksana}

\author{
Kharkiv National Agrarian University named after V.V.Dokuchayev, Ukraine \\ Corresponding E-mail: OksanaMandraji23@list.ru
}

Keywords: students' research activity, geometric fractals, properties of the Sierpinski carpet and the Menger sponge.

\begin{abstract}
This article describes the process of research of the properties of geometric fractals by high school students. The general formulas of calculating the length and the area of the Sierpinski carpet have been derived in the article. The total surface area and the volume of the Menger sponge have been calculated in the paper. The amazing facts of geometric fractals have been revealed. For instance, if $n \rightarrow \infty$, the length of the Sierpinski carpet is $L_{n} \rightarrow \infty$, and its area is $S_{n} \rightarrow 0$, similarly, if $n \rightarrow \infty$, the total surface area of the Menger sponge is $S_{t s(\text { п) }} \rightarrow \infty$, and its volume is $V_{n} \rightarrow 0$.
\end{abstract}

\section{INTRODUCTION}

Fractals are phenomena which became the part of the scientific world relatively recently, only in last quarter of the $20^{\text {th }}$ century. Since then, the interest to them has not disappeared, as a fractal can be a model of complex natural systems. Besides, due to its fascinating beauty and amazing properties, it can become inexhaustible material for organization of students' research activity.

\section{LITERATURE REVIEW}

Benoit Mandelbrot, a mathematician who first used the term "fractal", wrote: "I coined the word "fractal" based on Latin adjective "fractus" that means irregular, recursive, fragmented" [1]. There is no final determination of a fractal in Mathematics (in general, it is considered to be so), but search continues, that is why we give definitions which are appropriate for this article. A fractal is a self-similar structure whose display does not depend on the scale; it is a recursive model, each part of which repeats the development of the entire model in its own development $[2,3,4]$. Among all types of fractals the visual fractals are geometric fractals. The history of fractals started with them. Fractals of this type are got by simple geometric constructions, that is why they are simple for students and can inspire them on search and own developments.

\section{METHODOLOGY}

For this research it was decided to consider geometrical fractals "the Sierpinski carpet" and "the Menger sponge" in order to determine how the length, the area and the volume of figures change depending on step $n$ of recursion and if $n$ tends to infinity. When investigating the properties of the mentioned fractals, it is necessary to take into consideration that students will need special skills and abilities to find and determine consistent pattern (regularity), master the method of mathematical induction, knowledge on a geometric progression (also known as a geometric sequence) and the limit of a sequence.

\section{DISCUSSION AND RESULTS}

The Sierpinski carpet can be constructed in the following way: you should take a square with sides equal to units of measurement; after that each side of the square is divided into three equal parts, and accordingly the square is divided into nine equal squares with a side equal to $\frac{1}{3}$. A 
central square is cut out of the resulting figure. After that, the same thing is done with each of the eight squares remained, etc. (Fig. 1).

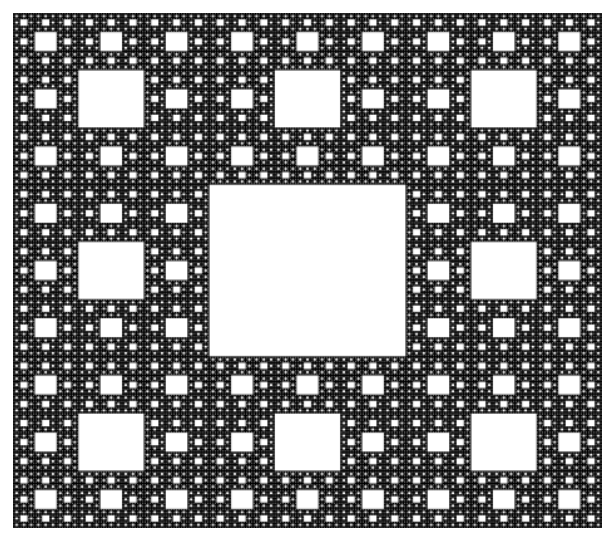

Fig. 1

Suppose a side of the Sierpinski carpet to be equal to $a$, then the length of curve, that limits it, is $L_{0}=4 a$. After the first step when the mean square with a side equal to $\frac{1}{3} a$, is cut out, the length of curve $L_{1}$, which limits the area remained, is $L_{1}=4 a+4 \cdot \frac{a}{3}=4 a\left(1+\frac{1}{3}\right)$. Reasoning similarly, at the second step we have $L_{2}=4 a+4 \cdot \frac{a}{3}+8 \cdot 4 \frac{a}{9}=4 a\left(1+\frac{1}{3}+\frac{8}{9}\right)$, on the third step we have $L_{3}=4 a+4 \cdot \frac{a}{3}+8 \cdot 4 \cdot \frac{a}{9}+8^{2} \cdot 4 \cdot \frac{a}{27}=4 a\left(1+\frac{1}{3}+\frac{8}{3^{2}}+\frac{8^{2}}{3^{3}}\right)$. Having taken a closer look, a certain consistent pattern can be noticed, so we can surmise that

$L_{n}=4 a+4 \cdot \frac{a}{3}+8 \cdot 4 \cdot \frac{a}{9}+8^{2} \cdot 4 \cdot \frac{a}{27}+\cdots+8^{n-1} \cdot 4 \cdot \frac{a}{3^{n}}=4 a \cdot\left(1+\frac{1}{3}+\frac{8}{3^{2}}+\frac{8^{2}}{3^{3}}+\cdots+\frac{8^{n-1}}{3^{n}}\right) . \quad$ The correctness of this assumption can be easily proved by the method of mathematical induction on the basis of belonging of the figure to self-similar figures. Thus, starting with $n \geq 2$, we have: if $n=2, \quad L_{2}=4 a\left(1+\frac{1}{3}+\frac{8}{9}\right)$, so the formula is true. Suppose $n=k$, then $L_{k}=4 a \cdot\left(1+\frac{1}{3}+\frac{8}{3^{2}}+\right.$ $\left.\frac{8^{2}}{3^{3}}+\cdots+\frac{8^{k-1}}{3^{k}}\right)$ is also the correct equality, and we prove the correctness of the derived formula for $n=k+1$, i.e. we need to get $L_{k+1}=4 a \cdot\left(1+\frac{1}{3}+\frac{8}{3^{2}}+\frac{8^{2}}{3^{3}}+\cdots+\frac{8^{k-1}}{3^{k}}+\frac{8^{k}}{3^{k+1}}\right)$. In fact, in order to get the length of the Sierpinski carpet at the step $k+1$, the length of all the squares which were added at the next step should be added to the length of step $k$. Starting with the second step, the quantity of squares which are formed is 8 in an appropriate degree, and as a result we have $L=4 a$. $\left(1+\frac{1}{3}+\frac{8}{3^{2}}+\frac{8^{2}}{3^{3}}+\cdots+\frac{8^{k-1}}{3^{k}}\right)+4 a \cdot \frac{8^{k}}{3^{k+1}}=4 a \cdot\left(1+\frac{1}{3}+\frac{8}{3^{2}}+\frac{8^{2}}{3^{3}}+\cdots+\frac{8^{k-1}}{3^{k}}+\frac{8^{k}}{3^{k+1}}\right), \quad$ quod erat prove.

Let us consider the derived formula again:

$L_{0}=4 a$

$L_{1}=4 a+\frac{4 a}{3}=4 a\left(1+\frac{1}{3}\right)$

$L_{2}=4 a+\frac{4 a}{3}+8 \cdot 4 \frac{a}{9}=4 a\left(1+\frac{1}{3}+\frac{8}{9}\right)$

$L_{3}=4 a+\frac{4 a}{3}+\frac{32 a}{9}+8^{2} \cdot 4 \cdot \frac{a}{27}=4 a\left(1+\frac{1}{3}+\frac{8}{3^{2}}+\frac{8^{2}}{3^{3}}\right)$

$L_{n}=4 a+4 \cdot \frac{a}{3}+8 \cdot 4 \cdot \frac{a}{9}+8^{2} \cdot 4 \cdot \frac{a}{27}+\cdots+8^{n-1} \cdot 4 \cdot \frac{a}{3^{n}}=4 a\left(1+\frac{1}{3}+\frac{8}{3^{2}}+\frac{8^{2}}{3^{3}}+\cdots+\frac{8^{n-1}}{3^{n}}\right)$

In the brackets, starting from the second summand, we have a sum of geometric progression, where $b_{1}=\frac{1}{3}$ and $q=\frac{8}{3}$, which can be found by the formula: $S_{n}=\frac{b_{1}\left(1-q^{n}\right)}{1-q}$. 


$$
S_{n}=\frac{\frac{1}{3}\left(1-\left(\frac{8}{3}\right)^{n}\right)}{1-\frac{8}{3}}=\frac{1-\left(\frac{8}{3}\right)^{n}}{-5}
$$

If $n$ tends to infinity, we have $L_{n}=4 a \cdot\left(1-\frac{1-\left(\frac{8}{3}\right)^{n}}{5}\right)=4 a \cdot \frac{4-\left(\frac{8}{3}\right)^{n}}{5}$

$\lim _{n \rightarrow \infty} L_{n}=\lim _{n \rightarrow \infty} 4 a \cdot \frac{4-\left(\frac{8}{3}\right)^{n}}{5}=4 a \lim _{n \rightarrow \infty} \frac{4-\left(\frac{8}{3}\right)^{n}}{5}=4 a \lim _{n \rightarrow \infty} \frac{\frac{4}{\left(\frac{8}{3}\right)^{n}}-1}{\frac{5}{\left(\frac{8}{3}\right)^{n}}}=\infty$

Thus, we have derived a general formula of the length of curve, which limits the area of the Sierpinski carpet, and have determined that with unrestricted growth of steps (when $n \rightarrow \infty$ ) the length of this curve also increases unrestrictedly $L_{n} \rightarrow \infty$.

Now let us consider how to calculate the area of the Sierpinski carpet, how it changes at every step of recursion and what it tends to if $n \rightarrow \infty$. According to the considerations given above we can write:

$S_{0}=a^{2}$

$S_{1}=a^{2}-\frac{a^{2}}{9}=\frac{8 a^{2}}{9}$

$S_{2}=\frac{8 a^{2}}{9}-\frac{8 a^{2}}{81}=\frac{8}{9} a^{2}$

$S_{3}=\frac{64 a^{2}}{81}-8^{2} \frac{1}{27^{2}} a^{2}=\frac{(9 \cdot 64-64) a^{2}}{9^{3}}=\frac{8^{3} a^{2}}{9^{3}}$

Having taken a closer look, it can be admitted that $S_{n}=\frac{8^{n} a^{2}}{9^{n}}$, and the correctness of the formula can be proved by the method of mathematical induction, based on the fact that the Sierpinski carpet is a self-similar figure. So, if $n=1$, then $S_{1}=\frac{8 a^{2}}{9}$, which is correct. Suppose that $S_{k}=\frac{8^{k} a^{2}}{9^{k}}$ if $n=k n=k$, and let us prove the correctness of the formula for $n=k+1$ :

$$
S_{k+1}=S_{k}-\frac{8^{k} a^{2}}{\left(3^{k+1}\right)^{2}}=\frac{8^{k} a^{2}}{9^{k}}-\frac{8^{k} a^{2}}{9^{k} \cdot 9}=\frac{9 \cdot 8^{k} a^{2}-8^{k} a^{2}}{9^{k} \cdot 9}=\frac{8^{k} a^{2}(9-1)}{9^{k+1}}=\frac{8^{k+1} a^{2}}{9^{k+1}}
$$

Thus, using the method of mathematical induction it has been proved that the area of the Sierpinski carpet at every step can be found by the formula: $S_{n}=\frac{8^{n} a^{2}}{9^{n}}$.

If $n \rightarrow \infty$, then $\lim _{n \rightarrow \infty} S_{n}=\lim _{n \rightarrow \infty} a^{2} \cdot\left(\frac{8}{9}\right)^{n}=a^{2} \lim _{n \rightarrow \infty}\left(\frac{8}{9}\right)^{n}=0$.

As a result, we have found an amazing thing: if $n \rightarrow \infty$, then $L_{n} \rightarrow \infty$, and $S_{n} \rightarrow 0$. Now it seems to be interesting to learn some amazing things about the Menger sponge.

The Menger sponge (also known as the Menger universal curve) is a three-dimensional analogue of the Sierpinski carpet. It is constructed in the following way: a cube is divided into 27 equal cubes, and 7 mean cubes are cut out. The same thing is done with the cubes remained, etc. (Fig. 2). 


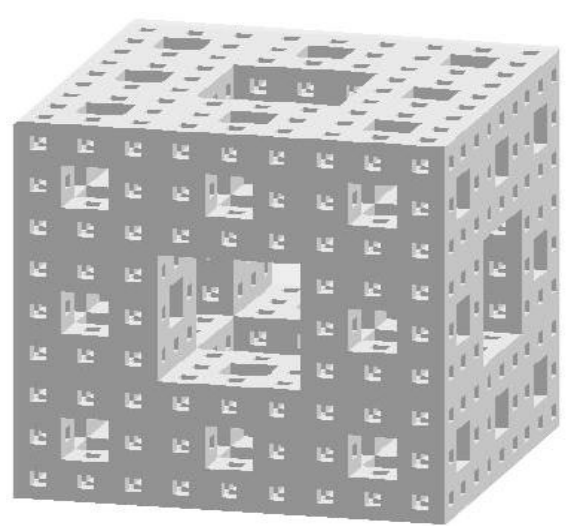

Fig. 2

In order to calculate the area of the Menger sponge, we should consider Fig. 3.

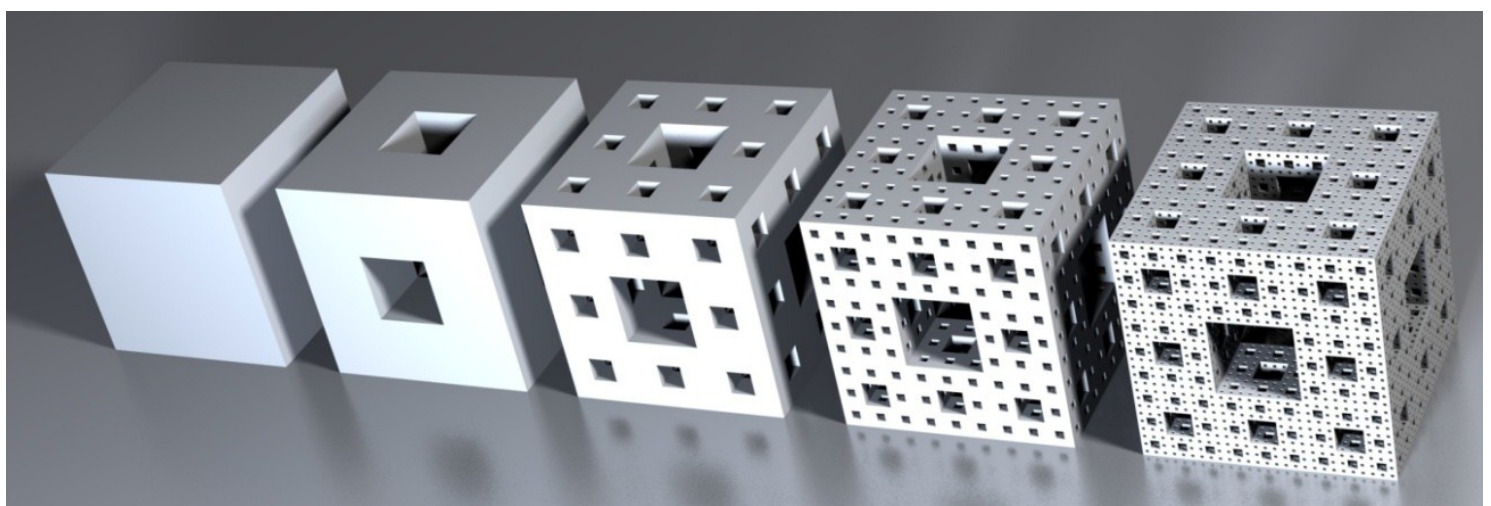

Fig. 3

As mentioned above, 7 cubes are cut out of the figure at the first step (Fig. 4), and this process continues recurrently. So, the total surface area at every step is:

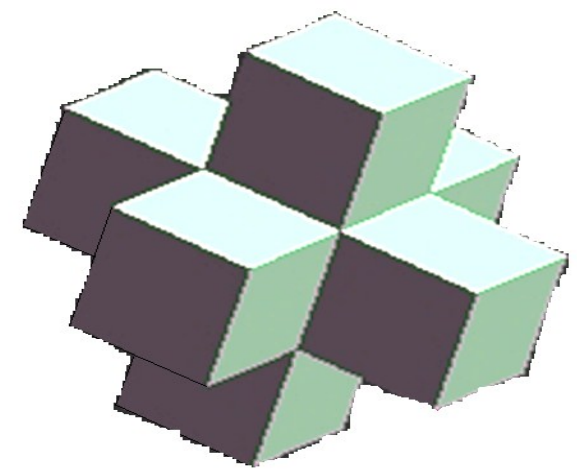

Fig. 4

$$
\begin{aligned}
S_{t s(0)}= & 6 a^{2} \\
S_{t s(1)}= & 6 a^{2}+6 \cdot 4\left(\frac{a}{3}\right)^{2}-6\left(\frac{a}{3}\right)^{2}=6 a^{2}+\frac{18 a^{2}}{9}=6 a^{2}\left(1-\frac{1}{9}+\frac{4}{9}\right) \\
S_{t s(2)} & =6 a^{2}\left(1-\frac{1}{9}+\frac{4}{9}\right)+20 \cdot 6 \cdot 4\left(\frac{a}{9}\right)^{2}-8 \cdot 6 \cdot\left(\frac{a}{9}\right)^{2}-4 \cdot 6 \cdot\left(\frac{a}{9}\right)^{2} \\
& =6 a^{2}\left(1-\frac{1}{9}+\frac{4}{9}-\frac{8}{9^{2}}+\frac{4 \cdot 19}{9^{2}}\right)
\end{aligned}
$$




$$
\begin{aligned}
S_{t s(4)}=6 a^{2}( & \left.1-\frac{1}{9}+\frac{4}{9}-\frac{8}{9^{2}}+\frac{4 \cdot 19}{9^{2}}-\frac{8^{2}}{27^{2}}+\frac{4 \cdot 19 \cdot 20}{27^{2}}\right)+20^{3} \cdot 6 \cdot 4\left(\frac{a}{81}\right)^{2}-8^{3} \cdot 6\left(\frac{a}{81}\right)^{2}-20^{2} \\
& \cdot 6 \cdot 4\left(\frac{a}{81}\right)^{2} \\
= & 6 a^{2}\left(1-\frac{1}{9}+\frac{4}{9}-\frac{8}{9^{2}}+\frac{4 \cdot 19}{9^{2}}-\frac{8^{2}}{27^{2}}+\frac{4 \cdot 19 \cdot 20}{27^{2}}-\frac{8^{3}}{81^{2}}+\frac{4 \cdot 19 \cdot 20^{2}}{81^{2}}\right)
\end{aligned}
$$

A certain consistent pattern can be noticed here, so we can write:

$$
\begin{aligned}
S_{t s(n)}=6 a^{2}( & 1-\frac{1}{9}+\frac{4}{9}-\frac{8}{9^{2}}+\frac{4 \cdot 19}{9^{2}}-\frac{8^{2}}{27^{2}}+\frac{4 \cdot 19 \cdot 20}{27^{2}}-\frac{8^{3}}{81^{2}}+\frac{4 \cdot 19 \cdot 20^{2}}{81^{2}}-\cdots-\frac{8^{n-1}}{3^{2 n}} \\
& \left.+\frac{4 \cdot 19 \cdot 20^{n-2}}{3^{2 n}}\right)
\end{aligned}
$$
induction.

The correctness of this formula can be easily proved by the method of mathematical

Having taken a closer look, we can simplify its general view. In brackets we have two geometric progressions $b_{n}: \frac{1}{9}, \frac{8}{9^{2}}, \frac{8^{2}}{27^{2}}, \frac{8^{3}}{81^{2}} \ldots$ and $c_{n}: \frac{4 \cdot 19}{9^{2}}, \frac{4 \cdot 19 \cdot 20}{27^{2}}, \frac{4 \cdot 19 \cdot 20^{2}}{81^{2}}, \ldots$ Let us find their sums: for $\left(b_{n}\right) b_{1}=\frac{1}{9}, q=\frac{8}{3^{2}}$, where $|q|<1$, thus, we have indefinitely declining geometric progression if $n \rightarrow \infty$, and the sum of it is $S_{n}=\frac{b_{1}}{1-q}=\frac{\frac{1}{9}}{1-\frac{8}{9}}=1$. For $\left(c_{n}\right) c_{1}=\frac{4 \cdot 19}{9^{2}}, q=\frac{20}{3^{2}}$ according to the formula $S_{n}=\frac{b_{1}\left(1-q^{n}\right)}{1-q}$ we have $S_{n}=\frac{\frac{4 \cdot 19}{9^{2}} \cdot\left(1-\left(\frac{20}{9}\right)^{n}\right)}{1-\frac{20}{9}}=-\frac{76}{99}\left(1-\frac{20^{n}}{9}\right)$

$$
\lim _{n \rightarrow \infty} S_{t s(n)}=\lim _{n \rightarrow \infty} 6 a^{2}\left(\frac{4}{9}+\frac{76}{81}-\frac{76}{99}+\left(\frac{20}{9}\right)^{n}\right)=\lim _{n \rightarrow \infty} 6 a^{2}\left(\frac{548}{891}+\left(\frac{20}{9}\right)^{n}\right)=\infty
$$

The result is that the general formula of the total surface area of the Menger sponge is $S_{t s(n)} \rightarrow \infty$ at limiting transition when $n \rightarrow \infty$.

Similarly to the previous considerations let us calculate the volume of the Menger sponge at the first step, at the second step, etc. for determining a consistent pattern and deriving a general formula.

$V_{0}=a^{3}$

$V_{1}=a^{3}-\frac{7 a^{3}}{27}=\frac{20 a^{3}}{27}$

$V_{2}=\frac{20 a^{3}}{3^{3}}-\frac{7 \cdot 20 a^{3}}{9^{3}}=20 a^{3}\left(\frac{1}{27}-\frac{7}{9^{3}}\right)$

$V_{3}=20 a^{3}\left(\frac{1}{27}-\frac{7}{9^{3}}\right)-\frac{20^{2} \cdot 7 a^{3}}{27^{3}}=20 a^{3}\left(\frac{1}{27}-\frac{7}{9^{3}}-\frac{20 \cdot 7}{27^{3}}\right)=20 a^{3}\left(\frac{1}{3^{3}}-\frac{7}{3^{6}}-\frac{20 \cdot 7}{3^{9}}\right)$

$V_{4}=20 a^{3}\left(\frac{1}{3^{3}}-\frac{7}{3^{6}}-\frac{20 \cdot 7}{3^{9}}-\frac{20^{2} \cdot 7}{3^{12}}\right)$

We can admit that

$V_{n}=20 a^{3}\left(\frac{1}{3^{3}}-\frac{7}{3^{6}}-\frac{20 \cdot 7}{3^{9}}-\frac{20^{2} \cdot 7}{3^{12}}-\cdots-\frac{20^{n-2} \cdot 7}{3^{3 n}}\right)$

So, we can easily prove the correctness of the derived formula using the method of mathematical induction.

Let us consider the expression written in the brackets and rewrite it in the following way:

$$
\frac{1}{3^{3}}-\left(\frac{7}{3^{6}}+\frac{20 \cdot 7}{3^{9}}+\frac{20^{2} \cdot 7}{3^{12}}+\cdots+\frac{20^{n-2} \cdot 7}{3^{3 n}}\right)
$$


We have geometric progression, where $b_{1=\frac{7}{3^{6}}}$, and $q=\frac{20}{3^{3}}$.

Inasmuch as $|q|<1$, then this geometric progression is indefinitely decreasing. Let us find its sum for $n \rightarrow \infty$. It is: $S_{n}=\frac{b_{1}}{1-q}=\frac{7}{3^{6} \cdot\left(1-\frac{20}{27}\right)}=\frac{1}{27}$.

Taking into consideration the result obtained, we have:

$$
\lim _{n \rightarrow \infty} V_{n}=20 a^{3}\left(\frac{1}{3^{3}}-\frac{7}{3^{6}}-\frac{20 \cdot 7}{3^{9}}-\frac{20^{2} \cdot 7}{3^{12}}-\cdots-\frac{20^{n-2} \cdot 7}{3^{3 n}}\right)=0
$$

So, the volume of the Menger sponge tends to zero if $n \rightarrow \infty$, and at the same time the total surface of this figure is $S_{t s(n)} \rightarrow \infty$. Here again we face an amazing discovery that differs from the common understanding.

\section{CONCLUSION}

It should be noted that the formulas $L_{n}, S_{n}$ for the Sierpinski carpet and $S_{t s(n)}, V_{n}$ for the Menger sponge have not been derived immediately, as it has been an interesting, amazing and (at the same time) hard work and a specific research. For instance, for calculating the length of curve that limits the area of the Sierpinski carpet, first all the calculations were brought to the end:

$L_{0}=4 a$

$$
\begin{aligned}
& L_{1}=4 a+\frac{4 a}{3}=\frac{16}{3} a \\
& L_{2}=4 a+\frac{4 a}{3}+8 \cdot 4 \frac{a}{9}=\frac{80}{9} a \\
& L_{3}=4 a+\frac{4 a}{3}+\frac{32 a}{9}+8^{2} \cdot 4 \cdot \frac{a}{27}=\frac{496}{27} a
\end{aligned}
$$

However, examining the results, certain consistent pattern in denominators of obtained fractions can be noticed, but the thing with numerators was different. Yet we have understood that according to the definition of a fractal the consistent pattern exists here, so it should be revealed. After all, experimentation on different recording of the obtained results has led to such kind of their arrangement that enabled to notice a consistent pattern, suggest a formula for general case and prove its correctness. The investigation on calculating the total surface area of the Menger sponge has also been interesting. In this case we had to remember about new surface, the area of which should be added at every following step; besides we had to consider that some surfaces, the area of which should be subtracted from the obtained results, emerge, as they have been cut out on those parts of the figure, the area of which we had already calculated. For instance, at first the formulas had such forms:

$$
\begin{aligned}
S_{t s(0)}= & 6 a^{2} \\
S_{t s(1)} & =6 a^{2}+6 \cdot 4\left(\frac{a}{3}\right)^{2}-6\left(\frac{a}{3}\right)^{2}=6 a^{2}+\frac{18 a^{2}}{9}=6 a^{2}\left(1+\frac{1}{3}\right) \\
S_{t s(2)} & =6 a^{2}\left(1+\frac{1}{3}\right)+20 \cdot 6 \cdot 4\left(\frac{a}{9}\right)^{2}-8 \cdot 3 \cdot\left(\frac{a}{9}\right)^{2}-12 \cdot 4 \cdot\left(\frac{a}{9}\right)^{2} \\
& =6 a^{2}\left(1+\frac{1}{3}\right)+6 a\left(20 \cdot 4 \cdot \frac{1}{9^{2}}-4 \frac{1}{9^{2}}-8 \frac{1}{9^{2}}\right)
\end{aligned}
$$

where the first subtrahend in $S_{t s(2)}$ was the quantity of squares on cubes in the tops of the Menger sponge, and they were cut out at the second step. The second subtrahend was all the squares which were cut out on the cubes that were not taken into account, so they did not belong to cubes situated in the tops. But the determined approach of calculation was considered difficult, so we wanted to find easier way. Thus, for calculating the total surface it has been decided at first to subtract the quantity of squares which emerged on the edges after the next step, then we had to subtract the quantity of squares which were cut out inside the figures. In this way, we have derived the formulas where consistent pattern can be easily noticed: 


$$
\begin{aligned}
S_{t s(0)}= & 6 a^{2} \\
S_{t s(1)} & =6 a^{2}+6 \cdot 4\left(\frac{a}{3}\right)^{2}-6\left(\frac{a}{3}\right)^{2}=6 a^{2}+\frac{18 a^{2}}{9}=6 a^{2}\left(1-\frac{1}{9}+\frac{4}{9}\right) \\
S_{t s(2)} & =6 a^{2}\left(1-\frac{1}{9}+\frac{4}{9}\right)+20 \cdot 6 \cdot 4\left(\frac{a}{9}\right)^{2}-8 \cdot 6 \cdot\left(\frac{a}{9}\right)^{2}-4 \cdot 6 \cdot\left(\frac{a}{9}\right)^{2} \\
& =6 a^{2}\left(1-\frac{1}{9}+\frac{4}{9}-\frac{8}{9^{2}}+\frac{4 \cdot 19}{9^{2}}\right)
\end{aligned}
$$

The study of the main properties of the Sierpinski carpet and the Menger sponge enables students to derive general formulas of calculating the length and area of the Sierpinski carpet, the total surface area and the volume of the Menger sponge. It also allows to prove the amazing facts, namely: if $n \rightarrow \infty$, then the length of the Sierpinski carpet is $L_{n} \rightarrow \infty$ and its area is $S_{n} \rightarrow 0$. Similarly, if $n \rightarrow \infty$, the total surface area of the Menger sponge is $S_{t s(\Pi)} \rightarrow \infty$ and its volume is $V_{n} \rightarrow 0$. Actually, the properties described are really interesting, that is why the world of fractals seems to be still full of secrets that can be studied subsequently, which is important for students' education.

\section{References}

[1] B. Mandelbrot, Fractal Geometry of Nature, The Institute of Computer Studies, Moscow, 2002.

[2] I. Sokolov. Fractals: submitted to the journal "Quantim" (1989), 5, pp. 6-13.

[3] E. Feder. Fractals, World, Moscow, 1991.

[4] O. Shkolniy. The Study of Elements of Fractal Theory at School: submitted to the journal "Mathematics at School" (2004), 9-10, pp. 42-47.

[5] Information on http://ru.wikipedia.org/wiki/Мандельброт,_Бенуа. 